ORIGINAL ARTICLE

\title{
Trauma: development of a sub-algorithm
}

\author{
W M Griggs, R W Morris, W B Runciman, G A Osborne*, A D Paix
}

Qual Saf Health Care 2005;14:e21 (http://www.qshc.com/cgi/content/full/14/3/e21). doi: 10.1136/qshc.2002.004499

*Dr Osborne died before this research was published.

See end of article for authors' affiliations

....................

Correspondence to:

Professor W B Runciman,

President, Australian

Patient Safety Foundation,

GPO Box 400, Adelaide,

South Australia, 5001,

Australia; research@

apsf.net.au

Accepted 12 January 2005
Background: Anaesthetists are regularly involved in the management of patients who have suffered trauma. Acute physiological derangements can occur at any time after the original injury, with life threatening sequelae. These problems may be complex in nature and evolve rapidly, often with an obscure aetiology, so a systematic approach to them is essential.

Objectives: To examine the role of a previously described core algorithm "COVER ABCD-A SWIFT CHECK" supplemented by a specific sub-algorithm for trauma, in the management of anaesthesia involving trauma cases.

Methods: The potential performance of a structured approach for each of the trauma incidents among the first 4000 incidents reported to the Australian Incident Monitoring Study (AIMS) was compared with the actual performance as reported by the anaesthetists involved.

Results: There were 38 relevant reports relating to trauma in the first 4000 reports to AIMS. In $39 \%$ of these there was "emergency corner cutting", although in the majority the urgency was thought to have been more perceived than real. The previously described "core" crisis management algorithm for crises during general anaesthesia was an effective means of discovering (82\%), diagnosing (68\%), and correcting $(66 \%)$ the majority of trauma incidents. However a sub-algorithm specific for the traumatised patient was required for unusual, obscure, or complex presentations.

Conclusion: Although the small numbers preclude validation of the sub-algorithm, it would have successfully managed all the trauma cases reported to AIMS.
E arly Management of Severe Trauma ${ }^{1}$ (EMST), also know as Advanced Trauma Life Support ${ }^{2}$ (ATLS), is a system which has been devised to systematically assess and resuscitate patients suffering from acute trauma. Many of these patients require anaesthesia and surgery, sometimes while still in the resuscitation phase of EMST, but also during the definitive care phase. Not infrequently, they will return to the operating theatre for months or even years. Given the now widespread acceptance of EMST it seems reasonable to extend the principles of EMST into the operating theatre when managing anaesthesia for acute trauma patients. EMST principles are also useful for resuscitation for a considerable time after the acute trauma episode, when close surveillance continues to be important. ${ }^{3}$ Acute physiological derangements can occur at any time after the original injury, with life threatening sequelae. These problems may be complex in nature and evolve rapidly, often with an obscure aetiology; so a systematic approach to them is essential.

In 1993, a "core" crisis management algorithm, represented by the mnemonic COVER ABCD-A SWIFT CHECK (the $\mathrm{AB}$ precedes COVER for the non-intubated patient), was proposed as the basis for a systematic approach to any crisis during anaesthesia where it is not immediately obvious what should be done, or where actions taken have failed to remedy the situation. ${ }^{4}$ This was validated against the first 2000 incidents reported to the original Australian Incident Monitoring Study (AIMS). AIMS is an ongoing study which involves the voluntary, anonymous reporting of any unintended incident which reduced, or could have reduced the safety margin for a patient. ${ }^{5}$

It was concluded that if this algorithm had been correctly applied, a functional diagnosis would have been reached within 40-60 seconds in 99\% of applicable incidents, and that the learned sequence of actions recommended by the COVER portion would have led to appropriate steps being taken to handle the $60 \%$ of problems relevant to this portion of the algorithm. ${ }^{4}$ However, this study also showed that the
$40 \%$ of problems represented by the remainder of the algorithm, ABCD-A SWIFT CHECK, were not always promptly diagnosed or appropriately managed..$^{4-6}$ It was decided that it would be useful, for these problems, to develop a set of specific sub-algorithms in an easy-to-use crisis management manual. ${ }^{7}$

This study reports on the place of the COVER ABCD-A SWIFT CHECK algorithm in the diagnosis and initial management of trauma, provides an outline of a specific crisis management sub-algorithm for crises arising in patients under anaesthesia for these problems, and provides an indication of the potential value of using this structured approach.

\section{METHODS}

Of the first 4000 reports to AIMS, those which made reference to "trauma" were extracted and analysed for relevance, causes, diagnosis, management, and outcome. The COVER ABCD-A SWIFT CHECK algorithm, as presented elsewhere in this set of articles, ${ }^{7}$ was applied to each relevant report to determine the stages at which the problem might have been diagnosed and to confirm that activating the COVER portion would have led to appropriate initial steps being taken. As patients suffering from trauma are not dealt with by this algorithm, a specific sub-algorithm was developed for these problems based on EMST principles (see fig l) and its putative effectiveness was tested against the reports.

The decision to develop a draft trauma algorithm before complete data analysis was taken because of the wide acceptance of EMST principles and was done in an attempt to validate the algorithm by the data analysis. EMST comprises four phases with the life threatening issues usually being addressed during the primary survey. ${ }^{1}$ This consists of five elements:

A - airway with cervical spine control;

$\mathrm{B}$ - breathing including ventilation and oxygenation; 


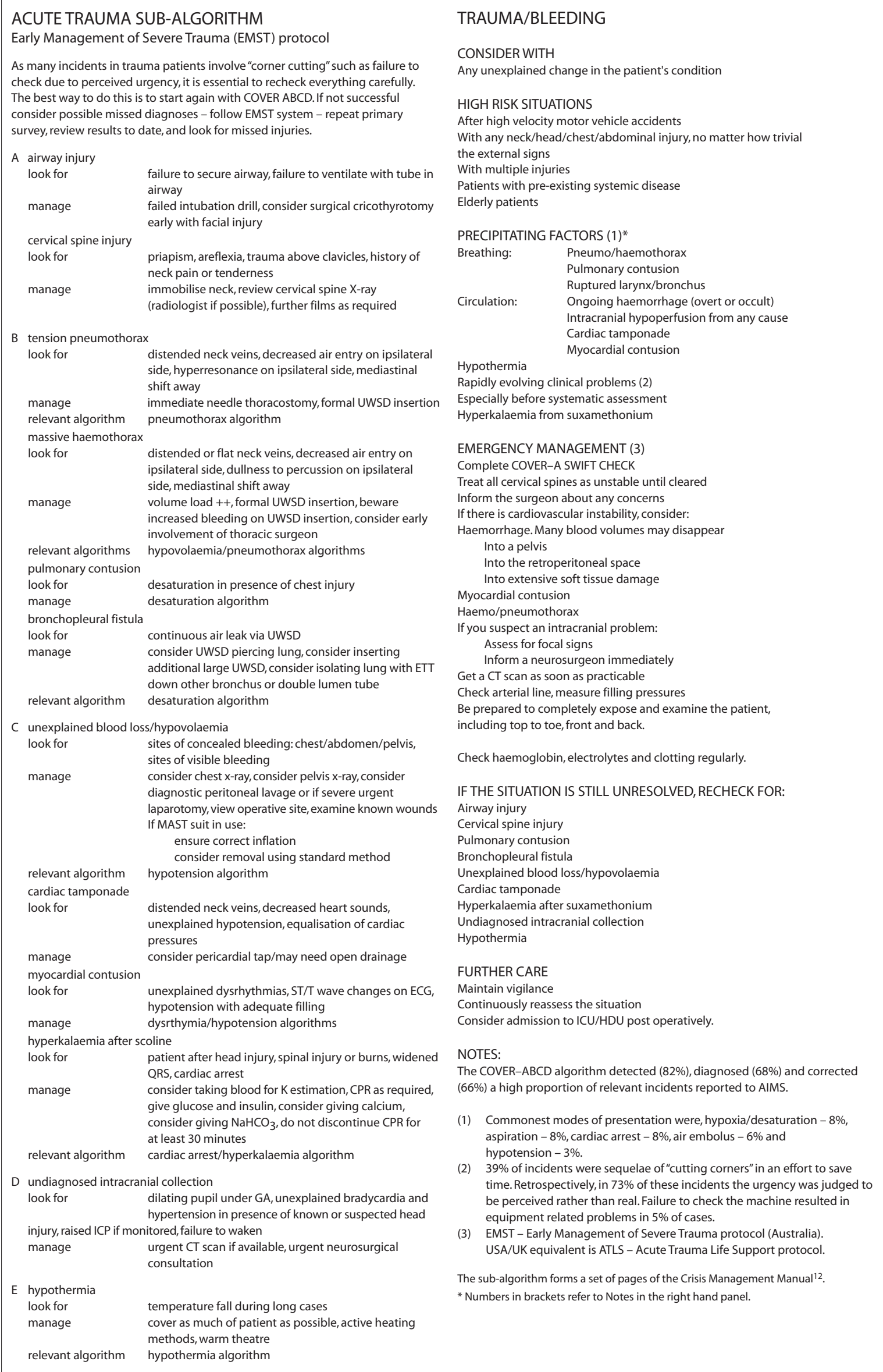

Figure 1 Acute trauma sub-algorithm. Early Management of Severe Trauma (EMST) protocol. 
Table 1 Classification according to the American Society of Anaesthesiologists (ASA) function/risk status

\begin{tabular}{llcr}
\hline ASA status & Definition & Number & \multicolumn{1}{l}{$\%$} \\
\hline I & Healthy patient & 11 & 29 \\
II & Mild systemic disease & 8 & 21 \\
III & Moderate systemic disease & 8 & 21 \\
IV & Severe systemic disease & 8 & 21 \\
V & Moribund patient & 3 & 8 \\
& Total & 38 & 100 \\
E & Emergency cases & 36 & 95 \\
\hline
\end{tabular}

C - circulation with haemorrhage control;

D - disability and pupil status (a neurological assessment); and finally,

E - exposure and environmental control.

The potential value of this structured approach (that is, the application of COVER ABCD-A SWIFT CHECK to the diagnosis and initial management of this problem, followed by the application of the sub-algorithm for management of trauma based on the EMST protocol) was assessed in the light of AIMS reports by comparing its potential effectiveness for each trauma incident with that of the actual management, as recorded in each report.

\section{RESULTS}

There were 87 reports that contained the word "trauma". Of these, 49 were either related to accidental trauma caused during anaesthesia to a non-trauma patient, most commonly damage to a tooth, or involved patients who had a past history of trauma mentioned in the report but which was unrelated to this episode. These unrelated cases were not used in the subsequent analysis and results. The remaining 38 reports related to patients with trauma where the trauma may have contributed to the incident or where the incident occurred in an acute trauma setting. Eight $(21 \%)$ of these 38 cases involved children 14 years or younger. Incidents were evenly spread across the range of ASA status (table 1) with a surprisingly high proportion (29\%) being ASA 1.

Hypoxia/desaturation was the clinical situation occurring most commonly with 20 instances (53\%). Fifteen incidents (39\%) in apparently urgent cases had "corner cutting" as a factor (table 2). In 11 of these 15 cases, further analysis revealed that the urgency was perceived rather than real.

The COVER ABCD core algorithm was useful in discovering $(82 \%)$, diagnosing $(68 \%)$, and correcting $(66 \%)$ of the trauma incidents (table 3 ).

A mixture of sub-algorithms including the proposed trauma sub-algorithm would have corrected the remaining incidents (table 4).

The breakdown of the specific incidents addressed by the trauma sub-algorithm (table 5) included three cases which

Table 2 Incidents related to "corner cutting"

\begin{tabular}{ll}
\hline Incident related to "corner cutting" & n (= 15) \\
\hline Ventilator/circuit failure due to no/poor checking & 6 \\
Temp set too high on humidifier & 1 \\
Partial extubation during movement & 2 \\
Throat pack left in & 1 \\
Missed oesophageal intubation & 1 \\
Uncrossmatched blood given & 1 \\
Nerve block wrong side & 1 \\
Suxamethonium given by mistake after intubation & 1 \\
Cl fracture missed pre-op-not viewed by radiologist & 1 \\
\hline
\end{tabular}

Table 3 Role of the various elements of COVER ABCD in discovering, diagnosing, and correcting incidents

\begin{tabular}{lccc}
\hline COVER ABCD Item & Discover & Diagnose & Correct \\
\hline Circulation (C1) & 4 & 1 & 1 \\
Colour (C2) & 9 & 3 & 0 \\
Oxygen (O1) & 0 & 1 & 4 \\
Oxygen analyser (O2) & 0 & 0 & 0 \\
Ventilate (V1) & 12 & 13 & 12 \\
Vaporizer (V2) & 0 & 0 & 0 \\
Endotracheal tube (E1) & 1 & 6 & 6 \\
Elimination (E2) & 0 & 0 & 1 \\
Review monitors (R1) & 1 & 1 & 0 \\
Review equipment (R2) & 1 & 1 & 1 \\
Airway (A) & 2 & NA & NA \\
Breathing (B) & 1 & NA & NA \\
Circulation (C) & 0 & NA & NA \\
Drugs (D) & 0 & NA & 0 \\
No success & 7 & 12 & 13 \\
Total number of cases & 38 & 38 & 38 \\
\hline
\end{tabular}

Table 4 Classification of reports according to the algorithm or sub-algorithm that definitively addresses the presenting incident

\begin{tabular}{lcc}
\hline Algorithm/sub-algorithm & $\mathbf{n}(\mathbf{= 3 8})$ & $\%$ \\
\hline COVER ABCD & 25 & 66 \\
Desaturation* & 9 & 24 \\
Trauma & 8 & 21 \\
Bronchospasm & 1 & 3 \\
Embolism & 2 & 5 \\
Hypothermia & 1 & 3 \\
Hyperthermia & 1 & 3 \\
\hline *All desaturation cases would have been addressed by COVER ABCD.
\end{tabular}

would have been amenable to prevention simply through better checking. These were a missed $\mathrm{Cl}$ fracture, a nerve block on the wrong side, and the giving of uncrossmatched blood. None of these incidents required any special corrective manoeuvres once they had been identified.

\section{DISCUSSION}

It is clear that anaesthetising the acutely injured patient can be very stressful. The high percentage of emergency ASA E codes (95\%) bears witness to this (table 1). The "emergency" situation seems to engender a sense of urgency that may then lead to "corner cutting" in an attempt to save time; 39\% of incidents seem to have occurred at least in part due to this "corner cutting" (table 2). For example, there seems to be a tendency to overlook checks in "emergency" trauma cases, when clearly these are the situations where one can least afford a failure of equipment. It is also crucial to note that on further analysis 11 of the 15 "corner cutting" cases were not

Table 5 Breakdown of incidents addressed by trauma sub-algorithm

\begin{tabular}{ll}
\hline Trauma sub-algorithm incident & $\mathbf{n ~ ( = 8 ) ~}$ \\
\hline Lacerated intrathoracic trachea & 1 \\
Broncho-pleural fistula & 1 \\
Hyperkalaemia after head injury & 2 \\
Uncrossmatched blood given* & 1 \\
C1 fracture missed pre-op* & 1 \\
Nerve block wrong side* & 1 \\
Hypotension due to incorrectly used MAST suit & 1 \\
\hline \multirow{2}{*}{ *Incident which can be addressed simply by being recognised. }
\end{tabular}




\section{Key messages}

- There were 38 reports relating to trauma in the first 4000 incidents reported to AIMS. Eight (21\%) of these involved children less than 14 years of age.

- These trauma cases related to incidents in which either the trauma may have contributed to the incident or the incident occurred in an acute trauma setting.

- Hypoxia/desaturation was the commonest occurring clinical incident (53\%).

- Fiffeen incidents (39\%) involved unjustified "corner cutting", where the level of urgency appeared to be more perceived than real. The commonest "corner cutting" incident was ventilation/circuit failure due to no/poor checking.

- ASA grades I to IV were almost equally represented among the 38 cases; 36 cases were emergencies, with three moribund cases.

- A specific trauma sub-algorithm was developed based on the widely accepted Early Management of Severe Trauma (EMST) course principles and its effectiveness was tested against the reports.

- The COVER ABCD core algorithm discovered $82 \%$, diagnosed $68 \%$, and corrected $66 \%$ of the trauma incidents.

- The application of several sub-algorithms from this series of papers, including the proposed trauma subalgorithm, would have corrected the remaining incidents in this trauma series. The sub-algorithms that definitively addressed the presenting incident were: desaturation in nine cases, trauma in eight cases, embolism in two cases, and bronchospasm, hypothermia, and hyperthermia in one case each.

- The single largest contributor to the successful correction of the trauma incidents was "ventilate by hand" (V1) in COVER.

- Although consistency with current EMST principles is important, a larger series of trauma incident reports is needed for ongoing validation and revision of the protocol.

shown to be time critical. The use of the ASA E code causes the patient to be labelled as either "elective" or "emergency". This "black and white" classification does not accord with reality, as there is clearly a spectrum of cases ranging from the true elective case to the true emergency. Thoughtful and careful attention to management may not be compatible with haste. ${ }^{8}$ A good example of this is acute trauma, where it is appropriate, even advantageous, to use a regional block for analgesia before surgical intervention. ${ }^{9}$

It is necessary for the anaesthetist to make considered judgements of the relative urgency and need for speed in each individual case. Even in those cases that are felt to be time critical, not taking the minimal time necessary to carry out routine equipment checks is at best a false economy and at worst extremely dangerous, as well as medico-legally indefensible.

As with the initial overall evaluation of the first 2000 incidents, ${ }^{4}$ the single largest contributor to the successful correction of incidents using COVER ABCD was "ventilate by hand" (VI); this would have corrected 32\% of incidents (table 3). The small numbers make it difficult to comment on the other elements of COVER ABCD, but $34 \%$ of trauma incidents would not have been addressed by COVER ABCD compared with $5 \%$ of such incidents in the original overall report. This again supports the view that trauma incidents have their own pattern and require the application of a specific trauma sub-algorithm. Given the small number of trauma cases, it is not possible to make much comment on the use of sub-algorithms in this $34 \%$ of the 38 trauma cases, other than to say that all cases would have been addressed if the trauma sub-algorithm had been used correctly (table 4).

EMST elements (fig 1, left panel) have been used to compose the acute trauma sub-algorithm by transposing them to the operating theatre environment. However a major issue in developing and testing the acute trauma subalgorithm has been the infrequency of reported trauma incidents that relate specifically to the injuries. Disregarding the failures of checking, there were only four problems identified (table 5). Accordingly although the proposed trauma sub-algorithm identifies a number of major trauma problems, it has not been possible to validate it by this review. The number of relevant cases is still too small and clearly many possible problems which have been reported previously in the literature have not been reported in these 4000 incident reports. Equally, rewriting the algorithm to address only the trauma incidents found would leave major gaps when compared with well recognised if apparently uncommon problems. For example, the analysis of pneumothoraces in the first 4000 cases $^{10}$ revealed that 24 were reported, with 17 associated with general anaesthesia, six with nerve blocks in the chest wall or clavicular regions, and one with an intercostal drain mishap, but although pneumothorax is a not uncommon trauma diagnosis, none were found among the trauma incidents reports. It is felt that it would be unwise to exclude pneumothorax from the trauma algorithm.

At this time, while the above data are insufficient to validate any trauma sub-algorithm, the sub-algorithm suggested (fig l) is consistent with EMST principles currently used for management of acute trauma in the field ${ }^{11}$ and has successfully addressed the few trauma cases in the data. It will be appropriate to wait for further reports with a view to ongoing validation and revision as necessary.

Finally, it is important that a full explanation of what happened be given to the patient and that the problem be clearly documented in the anaesthetic record. If a particular precipitating event was significant, or a particular action was useful in resolving the crisis, this should be clearly explained and documented.

\section{ACKNOWLEDGEMENTS}

The authors would like to thank all the anaesthetists in Australia and New Zealand who contributed to the 4000 incident reports upon which this and the other 24 papers in the Crisis Management Series are based. The coordinators of the project also thank Liz Brown for preparing the draft of the original Crisis Management Manual; Loretta Smyth for typing; Monika Bullock, RN, for earlier coding and classifying of data; Dr Charles Bradfield for the electronic version of the algorithms; Dr Klee Benveniste for literature research; Drs Klee Benveniste, Michal Kluger, John Williamson, and Andrew Paix for editing and checking manuscripts.

\section{Authors' affiliations}

W M Griggs, Director, Trauma Service, Royal Adelaide Hospital and University of Adelaide, Adelaide, South Australia, Australia

R W Morris, Director of Research and Development, Sydney Medical Simulation Centre, Royal North Shore Hospital, St Leonards, New South Wales, Australia

W B Runciman, Professor and Head, Department of Anaesthesia and Intensive Care, University of Adelaide and Royal Adelaide Hospital, Adelaide, South Australia, Australia 
G A Osborne, Senior Staff Specialist, Department of Anaesthesia and Intensive Care, Royal Adelaide Hospital and University of Adelaide, Adelaide, South Australia, Australia

A D Paix, Consultant Anaesthetist, Princess Royal University Hospital, Orpington, Kent, UK

This study was coordinated by The Australian Patient Safety Foundation, GPO Box 400, Adelaide, South Australia, 5001, Australia.

\section{REFERENCES}

1 Trauma Committee, Royal Australasian College of Surgeons. Early management of severe trauma. Box Hill, Victoria: Capitol Press, 1992.

2 Committee on Trauma, American College of Surgeons. Advanced Trauma Life Support. Chicago: American College of Surgeons, 1988.

3 Ingelmo $P$, Barone $M$, Fumagalli R. Importance of monitoring in high risk surgical patients. Minerva Anestesiol 2002;68:226-30.

4 Runciman WB, Webb RK, Klepper ID, et al. Crisis management: validation of an algorithm by analysis of 2000 incident reports. Anaesth Intensive Care 1993;21:579-92.
5 Webb RK, Currie M, Morgan CA, et al. The Australian Incident Monitoring Study: an analysis of 2000 incident reports. Anaesth Intensive Care 1993;21:520-8.

6 Webb RK, van der Walt JH, Runciman WB, et al. Which monitor? An analysis of 2000 incident reports. Anaesth Intensive Care 1993;21:529-42.

7 Runciman WB, Kluger MT, Morris RW, et al. Crisis management during anaesthesia: the development of an anaesthetic crisis management manual. Qual Saf Health Care 2005; 14:e1.

8 Bedell E, Prough DS. Anesthetic management of traumatic brain injury. Anesthesiol Clin North America 2002:20:417-39.

9 Calkins MD, Kuzma PJ, Larkin TM, et al. Pain management in the special operations environment: regional anesthetics. Mil Med 2001;166:211-16.

10 Bacon AK, Paix AD, Williamson JA, et al. Crisis management during anaesthesia: pneumothorax. Qual Saf Health Care 2005; 14:e18.

11 Griggs WM. Early assessment of the acute severe trauma patient. ADF Health 2001;2:4-11. Available at http://www.defence.gov.au/dpe/dhs/ infocentre/publications/journals/NoIDs/ADFHealthAprO1/ ADFHealthApr01 2 1 04-11.pdf (accessed 14 November 2004).

12 Australian Patient Safety Foundation. Crisis Management Manual: COVER $A B C D$ A SWIFT CHECK. Adelaide: Australian Patient Safety Foundation, 1996:74. Available at http://www.apsf.net.au/anaesthesia.htm (accessed 6 September 2004) 